\title{
Uma Abordagem baseada nas Preferências dos Docentes para o Problema de Programação de Horários em Universidades
}

\author{
Osmar Leandro Dantas da Silva ${ }^{1}$, Diego Rocha Lima ${ }^{1}$ \\ ${ }^{1}$ Instituto Federal do Ceará campus Crato (IFCE) \\ Caixa Postal 63115-500 - Crato - CE - Brasil \\ osmarleandrods@gmail.com, diego.rocha@ifce.edu.br
}

\begin{abstract}
The university timetabling problem is usually solved by the responsible manager on planning with the teaching staff of the institution. The preferences of teachers may not be relevant in certain respects, which does not guarantee they are always enforced. Thus, this work will approach the issue of allocation of time at universities, with the proposal of an accurate model. The optimal solutions of the tests were found in medium 38 minutes for the instance with 263.040 decision variables, which makes it feasible to use the model to generate the six-month timetable.
\end{abstract}

Resumo. O problema de programação de horários em universidades é resolvido normalmente pelo gestor responsável através de planejamento com a equipe pedagógica da instituição. As preferências dos docentes podem não ser relevantes em certos aspectos, o que não garante que sempre sejam obedecidas. Dessa forma, neste trabalho será abordado o problema de alocação de horários em universidades, com a proposta de um modelo exato. As soluções ótimas dos testes foram encontradas no periodo médio de 38 minutos para a instância com 263.040 variáveis de decisão, o que torna viável a utilização do modelo para gerar a grade horária semestral.

\section{Introdução}

A alocação de horários em universidades consiste na programação de docentes, disciplinas e seus respectivos horários semestrais, respeitando certas restrições. Algumas das restrições são quantidade de salas de aula e laboratórios, quantidade de docentes, dias letivos e disciplinas, Os recursos da instituição são alocados de forma a alcançar um objetivo pré-definido (BUCCO, 2014).

O responsável pela programação de horários geralmente realiza suas atividades manualmente, ou assistido por computador, alocando um recurso humano para cada aula em um horário. Atender as restrições do corpo docente e da coordenação institucional geralmente dificulta a obtenção de um horário sólido, uma vez que estas alterações podem influenciar em outros horários de forma encadeada.

Segundo Schaerf (1999), a instância deste problema geralmente possui milhares de variáveis, sendo que estas variáveis devem respeitar as restrições da própria instituição. Assim, o planejamento pode envolver dias, o que nem sempre torna viável uma programação de horários de forma satisfatória, o que justifica a busca por uma solução automatizada. 
Este trabalho tem como objetivo apresentar um modelo matemático que, considerando as preferências dos docentes por disciplinas, dias e horários, realize a alocação de horários de um curso superior.

\section{Trabalhos Relacionados}

O problema de escalonamento de horários é recorrente e bastante estudado. Diversas soluções para cenários distintos já foram apresentados e consolidados na literatura. Dessa forma, esta revisão tem como base trabalhos publicados entre 2005 e 2015, que tratam algum tipo de preferência acadêmica.

A solução desenvolvida por Hime (2015) consiste em um modelo matemático inteiro, em conjunto com uma interface web desenvolvida para interação com o usuário. A instância utilizada por ele foi construída com base nos cursos de Pós-graduação do Departamento de Estatística e Informática da UFRPE, que envolve 58 disciplinas e 30 professores. Neste trabalho, o autor permitiu que o corpo docente indicasse suas preferências via e-mail.

A solução proposta por Bucco (2014) contribuiu para a literatura através de uma revisão bibliográfica bastante completa de diversos trabalhos que envolvem o problema. Além disso, seu trabalho propõe um modelo linear que se aproxima bastante da realidade. O modelo do autor foi implementado utilizando o Microsoft Access 2007, para carregamento de dados, Microsoft Visual C++ 2010 Express, para implementação do modelo matemático, e o solver IBM ILOG CPLEX, para solução da instância.

Já o modelo de Costa et al. (2012) foi desenvolvido em programação inteira buscando maximizar as preferências dos professores do departamento de Engenharia de Produção da UFPB, onde os docentes indicariam seu benefício para cada disciplina do curso, semelhante ao que acontece no trabalho de Hime (2015). Com relação às restrições, os autores definem carga horária para os docentes.

Com relação ao trabalho de Souza Filho e Gomes (2009), o problema é retratado no Departamento de Tecnologias e Linguagens do Instituto Multidisciplinar da UFRRJ. Neste trabalho, os autores apresentam um modelo matemático que foi testado utilizando instâncias com 36 disciplinas, 18 professores e 8 salas por dia, em dois horários noturnos, resultando em 3.240 variáveis inteiras e 167 restrições.

\section{Modelagem do Problema}

O desenvolvimento do modelo teve como base os trabalhos de Bucco (2014), Hime (2015) e Souza Filho e Gomes (2009). Os dados utilizados no modelo são definidos matematicamente através de conjuntos e matrizes, e serão explanados a seguir.

$p \in P \quad$ conjunto de docentes.

$d \in D \quad$ conjunto de dias letivos, exemplo: segunda-feira, sábado.

$s \in S \quad$ conjunto de salas de aula, exemplo: sala 1, laboratório 1.

$t \in T \quad$ conjunto de disciplinas semestrais, exemplo: Cálculo Diferencial I.

$h \in H \quad$ conjunto de horários, exemplo: 07:00 às 09:20.

$\mathrm{CH}_{t} \quad$ conjunto de carga horária de cada t-ésima disciplina.

$T P_{p, t} \quad$ conjunto de preferências do p-ésimo docente para a t-ésima disciplina.

$D P_{p, d, h} \quad$ preferências do p-ésimo docente para o d-ésimo dia no h-ésimo horário.

$\mathrm{O}$ conjunto $\mathrm{CH}_{\mathrm{t}}$ indica a quantidade de aulas da t-ésima disciplina durante a semana, no caso deverá ser uma ou duas vezes, enquanto que os conjuntos $\mathrm{TP}_{\mathrm{p}, \mathrm{t}}$ e $\mathrm{DP}_{\mathrm{p}, \mathrm{d}, \mathrm{h}}$ 
representam as preferências por disciplinas e por dias/horários, respectivamente. Para as restrições, considere o modelo abaixo.

$$
\begin{array}{cl}
\text { maximize } \sum_{p \in P, d \in D, s \in S, h \in H, t \in T} x_{p d s h t} \times T P_{p t} \times D P_{p d h} \\
\sum_{t \in T, S \in S} x_{p d h s t} \leq 1 & \forall p \in P, d \in D, h \in H \\
\sum_{p \in P, t \in T} x_{p d h s t} \leq 1 & \forall d \in D, h \in H, s \in S \\
\sum_{S \in S} y_{s t} \leq 1 & \forall t \in T \\
\sum_{p \in P} z_{p t} \leq 1 & \forall t \in T \\
\sum_{h \in H} x_{p d h s t} \leq 1 & \forall p \in P, d \in D, s \in S, t \in T \\
x_{p d h s t} \leq y_{s t} \quad & \forall p \in P, d \in D, h \in H, s \in S, t \in T \\
\sum_{p d h s t} \leq z_{p t} \times c h_{t} \quad & \forall p \in P, t \in T \\
x_{p d h s t} & \in\{0,1\} \\
y_{s t} & \in\{0,1\} \\
z_{s t} & \in\{0,1\}
\end{array}
$$

A função objetivo em (01) tem como foco encontrar a solução ótima considerando o valor das preferências dos docentes. Dessa forma, espera-se que a alocação seja realizada buscando maximizar este o valor resultante. Na restrição (02) é definido que cada professor em um horário e um dia deve estar alocado apenas a uma disciplina em uma sala. Já na restrição (03), cada sala de aula deve estar alocada para apenas uma aula (professor/disciplina) em um dia e um horário. Concomitantemente, a restrição (04) indica cada disciplina deve ter suas aulas estar alocadas em apenas uma sala. A restrição (05) define a variável $\mathrm{zpt}$, onde cada disciplina deve ter suas aulas alocadas para, no máximo, um docente.

A restrição (06) mostra que a quantidade de aulas (professor/disciplina) por dia não deve ultrapassar um, permitindo que o modelo não realize a alocação consecutiva de aulas para uma mesma disciplina. Em seguida, a restrição (07) define a alocação de apenas uma sala para cada aula no mesmo instante, limitando a quantidade de aulas consecutivas à quantidade de salas. A restrição seguinte (08) tem como objetivo alocar todas as aulas de uma disciplina para um único docente, além de realizar o controle da carga horária da disciplina.

Por fim, as restrições (09), (10) e (11) representam o domínio das variáveis de decisão. Considere que $\mathrm{x}_{\text {pdhst }}$ deve assumir o valor 1 quando indicar a alocação do $\mathrm{p}$-ésimo professor à t-ésima disciplina, no h-ésimo horário do d-ésimo dia na s-ésima sala. Com isso, a variável deverá assumir o valor 0 caso contrário. Por conseguinte, as variáveis $\mathrm{y}_{\mathrm{st}}$ e $z_{p t}$ indicam respectivamente a alocação da s-ésima sala para a t-ésima disciplina e a alocação do p-ésimo docente à t-ésima disciplina.

\section{Estudo de Caso e Resultados}

Para realizar os testes, foram utilizadas duas instâncias de tamanhos diferentes, sendo que uma delas contém os dados reais das preferências coletados com os docentes através da 
tecnologia Formulários Google. Já a máquina utilizada continha um processador Intel Core i7 3612QM (3,1Ghz), 8GB de memória RAM DDR3 e sistema operacional Linux Ubuntu 15.10 de 64 bits e AMPL for Students com o Gurobi 6.5. Antes de iniciar os testes com a instância da pesquisa em (P1), foi utilizada uma outra instância (P2) com a finalidade de testar a capacidade do modelo.

Tabela 1. Resultados dos testes por instância.

\begin{tabular}{c|c|c|c|c|c} 
Instância & Docentes & Disciplinas & Salas & Total de Variáveis & Tempo Resolução \\
\hline P1 & 15 & 43 & 08 & 45.623 & 6,59 segundos \\
\hline P2 & 25 & 80 & 13 & 263.040 & 39,3 minutos
\end{tabular}

O estudo de caso consistiu na coleta das preferências dos docentes do curso de Sistemas de Informação do Instituto Federal do Ceará campus Crato, por meio de uma pesquisa on-line realizada com Formulários Google. Como mostrado anteriormente, participaram 15 docentes, que informaram suas preferências sobre as 43 disciplinas, os cinco dias da semana e dois horários noturnos.

\section{Conclusão}

Pode-se concluir a partir do estudo de caso que a qualidade da alocação depende sumariamente da disponibilidade do corpo docente para as disciplinas do curso superior. No estudo, todas as disciplinas foram alocadas, já que existiam horários suficientes pra escalonar todo o curso. Concomitantemente, uma quantidade insuficiente de docentes ou de salas influência negativamente no resultado. Finalmente, conclui-se que o modelo pode substituir o método tradicional de programação de horário, permitindo tempo de resolução abaixo de 38 minutos. Esse tempo é aceitável uma vez que apresenta ganho de tempo comparando-se com o método tradicional. Como trabalhos futuros pretende-se criar uma aplicação web e disponibilizar para os outros cursos da instituição.

\section{Referências}

Bucco, G. B. (2014) "Construção de um modelo de programação linear para o University Timetabling Problem”. 156 f. Dissertação (Mestrado em Administração) Universidade Federal do Rio Grande do Sul. Porto Alegre, jun. 2014.

Costa, L. C. A.; Acioli, L. F.; Subramanian, A. (2012) "Um modelo de programação inteira para o problema de alocação de professores a turmas do departamento de engenharia de produção de uma instituição de ensino superior”. XLIV Simpósio Brasileiro de Pesquisa Operacional, Rio de Janeiro.

Hime, R. (2015) "Uma aplicação da Programação Inteira no School Timetabling Problem". Universidade Federal Rural de Pernambuco.

Schaerf, A. (1999) A survey of automated timetabling. In Artificial intelligence review, v. 13 , n. 2, p. 87-127.

Souza Filho, E. M.; Gomes, C. R. (2009) "Programação do quadro de horários de disciplinas de uma universidade via programação inteira". In XLI Simpósio Brasileiro de Pesquisa Operacional, Porto Seguro, 357-368.

Neukirchen, F. V. P. et al. (2014) "Um estudo de caso sobre a geração de quadros de horários no departamento de Ciência da Computação da UFRGS”. XLIV Simpósio Brasileiro de Pesquisa Operacional, Salvador - BA, p. 3272-3279. 